\title{
Evaluation of Serum Lysophosphatidic Acid and Lysophosphatidylcholine Levels in Major Depressive Disorder Patients
}

Sumaia Riya ${ }^{1}$, Sharmin Sultana ${ }^{1}$, Sohel Daria ${ }^{1}$, Maliha A. Proma ${ }^{1}$, Mohiuddin Ahmed Bhuiyan ${ }^{1}$, Md Ahsanul Haque ${ }^{1}$, Md Rabiul Islam ${ }^{1}$

1. Department of Pharmacy, University of Asia Pacific, Dhaka, BGD

Corresponding author: Md Rabiul Islam,robi.ayaan@gmail.com

\section{Abstract \\ Background}

Major depressive disorder (MDD) is a heterogeneous condition featured with a continuous low mood, feeling of sadness, lack of interest to perform daily activities. Genetic, physiological, biological, social, and environmental factors are associated with the pathophysiology of depression. Though several human studies failed to identify the suitable biological markers for depression, some animal studies showed phospholipids play a vital role in the alteration of emotion. Thus, the current study aimed to measure the serum levels of lysophosphatidic acid (LPA) and lysophosphatidylcholine (LPC) in MDD patients and healthy controls (HCs) to explore their roles and relationship with depression.

\section{Methods}

This case-control study enrolled 53 MDD patients and 50 HCs matched by age, gender, and body mass index. Based on the diagnostic and statistical manual of mental disorders, $5^{\text {th }}$ edition, a qualified psychiatrist diagnosed patients and assessed HCs. We applied the Hamilton depression rating scale (Ham-D) to measure the severity of depression. We used enzyme-linked immunosorbent assay kits to measure serum lysophosphatidic acid and lysophosphatidylcholine levels.

\section{Results}

We found no alterations of these parameters in serum levels of MDD patients compared to HCs. We also observed a significant positive correlation between LPA and LPC levels in MDD patients. Moreover, the present study showed no significant associations between target markers and either diagnosis of depression or Ham-D scores, or management of depression.

\section{Conclusion}

The present study suggests that LPA and LPC levels probably would not serve as potential biomarkers of MDD. Thus, we recommend further studies with large and more homogeneous populations to explore the

Review began 12/17/2020 Review ended 12/25/2020 Published 12/30/2020

\section{() Copyright 2020}

Riya et al. This is an open access article distributed under the terms of the Creative Commons Attribution License CC-BY 4.0., which permits unrestricted use, distribution, and reproduction in any medium, provided the original author and source are credited. exact relationship between serum lipids and MDD.

Categories: Neurology, Pathology, Psychiatry

Keywords: lysophosphatidic acid, lpa, lysophosphatidylcholine, lpc, major depressive disorder, mdd

\section{Introduction}

Major depressive disorder (MDD) is a complex psychiatric condition; it is unlikely to identify a single causal factor for it [1]. Genetic, biological, psychological, and environmental issues are involved in depression [2-6]. As MDD is highly comorbid, the neurobiological basis of depression needs to be known for efficient diagnosis and treatment [7]. Previous studies suggest the malfunction in neuronal proteins and peptides are involved in the pathophysiology of MDD [8-10]. Brain tissue and neural membrane contain a relatively high amount of glycerophospholipids [11]. These glycerophospholipids act as signaling molecules and are responsible for the formation of cell membranes [12]. As signaling molecules, they can easily promote anxiety and depressive disorders [7]. Lysophosphatidic acid (LPA) is one of the most significant reactive phospholipid species [13]. LPA is synthesized and metabolized in a large number of metabolic pathways. Among all, the enzymatic action of autotoxin is the most crucial pathway [14]. The second important pathway is to obtain LPA from membrane phospholipids with the help of phospholipase enzymes [15]. There are many other additional pathways for producing LPA, particularly by acyl-transferase enzymes [16]. MDD is one of the most common psychiatric illness that has created an immense burden in the community. For clinical purposes, there are no established biochemical markers for this disorder though LPA is a potent bioactive lipid mediator with various biological characteristics [17, 18]. The LPA1-receptor knockout mice showed altered emotional behavior due to the change of LPA receptors [19]. Therefore, LPA is one of the essential 
Lysophosphatidylcholine (LPC) is another kind of phospholipid that has a significant role in the neuronal mechanisms underlying the pathophysiology of several psychiatric disorders [20]. As a component of serum lipoprotein, LPC is a water-soluble amphiphilic lysophospholipid molecule [21]. In cell-biology, destabilization of the lysosomal process is troublesome for organelle and living cells [22]. LPC is one of the leading phospholipids elements of oxidized low-density lipoprotein (Ox-LDL) produced from the biochemical transformation of phosphatidylcholine [23]. The promptness of diverse signaling pathways is attached to the production of oxidative stress and inflammatory responses by LPC [24]. Studies suggest that polyunsaturated fatty acids could involve in the pathophysiology of MDD [25]. Based on the above findings, LPA and LPC might impact brain functioning and emotional behaviors. The role of LPA and LPC in depression is yet unclear though many studies conducted in these regards. Different researchers have different opinions regarding the association of LPA and LPC with depression [26, 27]. Therefore, the present study aimed to analyze serum LPA and LPC levels in MDD patients and their corresponding controls to decide the affiliation between the changed LPA and LPC levels and the seriousness of MDD.

\section{Materials And Methods}

\section{Study population}

To achieve a confidence level of $95 \%$ with a $\pm 5 \%$ margin of error, we needed to recruit 87 participants if the population proportion is $6 \%$. The present study included 103 subjects (53 MDD patients and 50 controls) for this case-control study based on the above assumption from September 2019 to July 2020. The patients enrolled from Bangabandhu Sheikh Mujib Medical University (BSMMU), Dhaka, Bangladesh. We recruited the matched (age, gender, and BMI) healthy controls (HCs) from the different locations of Dhaka city. A specialized psychiatrist diagnosed and evaluated both the cases and controls according to the diagnostic and statistical manual of mental disorders, 5th edition (DSM-5). All the participants performed a detailed neurological and physical screening for the coexistence of other complications. The severity scores of depression were measured using the 17-items Hamilton depression rating scale (Ham-D). Ham-D items were put together into the following segments: core (items 1, 2, 7, 8, 10,13), sleep (items 4, 5, 6), activity (items 7,8 ), psychic anxiety (items 9, 10), somatic anxiety (items 11,12, 13), delusion (items 2, 15) [28]. We excluded the subjects with a previous history of cardiovascular disease, epilepsy, hypertension, liver or kidney failure, and patients treated with any medication that could potentially modify the concentration of serum LPA and LPC levels. Also, we eliminated the patients who were suffering from mental retardation and other comorbid psychiatric illnesses. Additional exclusion criteria were participants with substance abuse or dependency, severe organic conditions, abnormal body mass index (BMI), and the presence of infectious diseases. We used a structured-predesigned questionnaire to record the socio-demographic and different biophysical characteristics.

\section{Blood sample collection and processing}

After overnight fasting of 10-12 hours, a professional phlebotomist collected blood samples (5mL) of study participants from the cephalic vein by using a plastic syringe fitted with a stainless-steel needle. The collected blood samples were placed into falcon tubes and were allowed to clot for an hour at room temperature without any agitation. The serum samples were extracted from the collected blood samples by centrifugation at $1000 \mathrm{x}$ g for 15 minutes at room temperature. We stored the separated serum samples immediately at $-80^{\circ} \mathrm{C}$ until being used for further analysis.

\section{Quantification of serum LPA and LPC}

We used commercially available enzyme-linked immunosorbent assay (ELISA) kits (Abbexa Ltd., UK) to quantify the serum LPA and LPC levels. That is a competitive binding ELISA technology where antibodies specific to LPA and LPC were pre-coated onto the 96 well-plates. Briefly, we took $50 \mu \mathrm{L}$ of standard solution and serum sample on a 96 well-plate and gently shaken the plate to mix the contents thoroughly. A $50 \mu \mathrm{L}$ of detection reagent-A was added to each well and gently shaken the plates for proper mixing. The plates were then sealed and incubated at $37^{\circ} \mathrm{C}$ temperature for 1 hour. Then discarded the liquid and washed the plates properly using wash buffer. After that, $100 \mu \mathrm{L}$ of detection reagent B was dispensed into each well with gentle mixing and sealed the plates for $30 \mathrm{~min}$ incubation at $37^{\circ} \mathrm{C}$. After the repeated washing process, added a $90 \mu \mathrm{L}$ tetramethylbenzidine (TMB) substrate to each well. The plates were incubated again for 15 $\min$ at $37^{\circ} \mathrm{C}$. Finally, we stopped the reaction by adding a $50 \mu \mathrm{L}$ of stop solution and took the absorbances immediately at $450 \mathrm{~nm}$. From these absorbance value, we calculated the serum LPA and LPC levels and presented as $\mu \mathrm{mol} / \mathrm{L}$. There was no cross-reactivity with other neurotrophic mediators. The coefficient of variance $(\mathrm{CV})$ for intra-assay and inter-assay was $<10 \%$ and $<12 \%$, respectively. The assay sensitivity for serum LPA and LPC were $<52.7 \mathrm{ng} / \mathrm{mL}$ and $<92.4 \mathrm{ng} / \mathrm{mL}$, respectively.

\section{Statistical analysis}

We carefully checked and verified all the collected data to detect errors and rectified them accordingly. Then we performed the coding, classification, and tabulation of data. We applied the independent sample t-test for the continuous variables and Fisher's exact test and categorical variables. We also conducted descriptive 


\section{Cureus}

statistics for the socio-demographic characteristics of the study participants. In this research, we used the box plot and scatter plot graphs to showcase the study findings. We applied the statistical package for social sciences (SPSS), version 25.0 for dada analyses (IBM Inc., Armonk, USA). We considered statistically significant results with p-values less than 0.05 .

\section{Results}

We observed that both the cases and controls were similar in terms of their descriptive information, as presented in Table 1.

\begin{tabular}{|c|c|c|c|}
\hline Variables & MDD patients $(n=53)$ mean \pm SEM & Healthy controls $(n=50)$ mean \pm SEM & p-value \\
\hline Age & $31.25 \pm 1.20$ & $34.53 \pm 1.60$ & 0.109 \\
\hline $18-24$ & $16(30 \%)$ & $11(22 \%)$ & \\
\hline $25-34$ & $17(32 \%)$ & $23(46 \%)$ & \\
\hline $35-44$ & $14(27 \%)$ & $13(26 \%)$ & \\
\hline 45-60 & $6(11 \%)$ & $3(6 \%)$ & \\
\hline Gender & & & 0.472 \\
\hline Male & $22(41 \%)$ & $17(34 \%)$ & \\
\hline Female & $31(59 \%)$ & $33(66 \%)$ & \\
\hline BMI $\left(\mathrm{kg} / \mathrm{m}^{2}\right)$ & $24.15 \pm 0.47$ & $25.66 \pm .073$ & $0 . .263$ \\
\hline Below 18.5 (CED) & $4(8 \%)$ & $2(4 \%)$ & \\
\hline 18.5-25 (normal) & $34(64 \%)$ & $27(54 \%)$ & \\
\hline Above 25 (obese) & $15(28 \%)$ & $21(42 \%)$ & \\
\hline Monthly income (KBDT) & $57.27 \pm 2.47$ & $60.35 \pm 3.25$ & 0.462 \\
\hline Below 30 & $3(6 \%)$ & $5(10 \%)$ & \\
\hline $30-60$ & $23(43)$ & $18(36 \%)$ & \\
\hline 61-90 & $19(36)$ & $12(24 \%)$ & \\
\hline Above 90 & $8(15)$ & $15(30 \%)$ & \\
\hline Education level & & & 0.237 \\
\hline Illiterate & $1(2 \%)$ & $5(10 \%)$ & \\
\hline Primary level & $6(11 \%)$ & $1(2 \%)$ & \\
\hline Secondary level & $29(55 \%)$ & $28(56 \%)$ & \\
\hline Graduate and above & $17(32 \%)$ & $16(32 \%)$ & \\
\hline Occupation & & & 0.253 \\
\hline Service & $9(17 \%)$ & $4(8 \%)$ & \\
\hline Business & $4(8 \%)$ & $8(16 \%)$ & \\
\hline Jobless & $7(13 \%)$ & $9(18 \%)$ & \\
\hline Others & $33(62 \%)$ & $29(58 \%)$ & \\
\hline Economic status & & & 0.332 \\
\hline Low & $13(25 \%)$ & $13(26 \%)$ & \\
\hline Medium & $34(64 \%)$ & $27(54 \%)$ & \\
\hline High & $6(11 \%)$ & $10(20 \%)$ & \\
\hline smoking nabit & & & 0.526 \\
\hline
\end{tabular}




\section{Cureus}

Yes

$11(21 \%)$

42 (79\%)
$9(18 \%)$

41 (82\%)

\section{TABLE 1: Socio-demographic characteristics of the study population}

MDD - major depressive disorder; BMI - body mass index; CED - chronic energy deficiency; KBDT - kilo Bangladeshi taka; SEM - standard error mean

According to the present study, most of the MDD patients were females with normal BMI. MDD patients and their comparing controls were alike with regard to age (patients: $31.25 \pm 1.20$, controls: $34.53 \pm 1.60$, $p=0.109$ ), BMI (patients: $24.15 \pm 0.47$, controls: $25.66 \pm 0.073$, $p=0.263$ ), and sex (male/female: $22 / 31,17 / 33$ patients and controls in a corresponding manner, $\mathrm{p}=0.472$ ). The non-smoker population with a medium economic impression was predominant in the present study. Following the past studies, the present study observed most of the MDD patients were in their third and fourth decades of life. Table 2 and Figure 1 demonstrated the clinical and laboratory findings of the study populations.

\begin{tabular}{|c|c|c|c|}
\hline Variables & MDD patients $(n=53)$ mean \pm SEM & Healthy controls $(n=50)$ mean \pm SEM & p-value \\
\hline Ham-D score & $15.90 \pm 0.57$ & $3.61 \pm 0.44$ & $<0.001$ \\
\hline Serum LPA ( $\mu \mathrm{mol} / \mathrm{L})$ & $0.59 \pm 0.09$ & $0.66 \pm 0.08$ & 0.543 \\
\hline Male & $0.58 \pm 0.15$ & $0.59 \pm 0.10$ & 0.991 \\
\hline Female & $0.60 \pm 0.12$ & $0.70 \pm 0.11$ & 0.552 \\
\hline Serum LPC $(\mu \mathrm{mol} / \mathrm{L})$ & $161.30 \pm 22.96$ & $167.59 \pm 22.16$ & 0.790 \\
\hline Male & $192.10 \pm 42.39$ & $149.72 \pm 42.39$ & 0.264 \\
\hline Female & $139.44 \pm 25.21$ & $176.79 \pm 21.82$ & 0.413 \\
\hline
\end{tabular}

\section{TABLE 2: Clinical characteristics and laboratory findings of the study population}

MDD - major depressive disorder; Ham-D - Hamilton depression rating scale; LPA - lysophosphatidic acid; LPC - lysophosphatidylcholine; SEM standard error mean
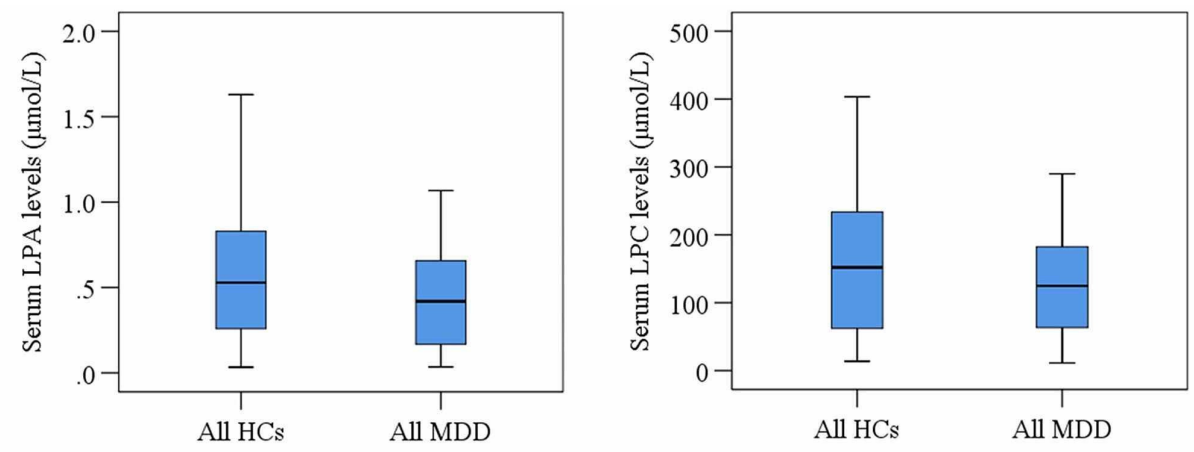

FIGURE 1: The box-plot graph depicts no significant changes in serum LPA and LPC among all of the study population between HCs and MDD patients

LPA - Iysophosphatidic acid; LPC - lysophosphatidylcholine; HCs - healthy controls; MDD - major depressive disorder

Serum LPA and LPC levels did not show any significant changes in MDD patients compared to HCs ( $p>0.05)$. Moreover, serum LPA and LPC concentrations did not correlate with age, gender, and BMI in the patient group (Figure 2). 

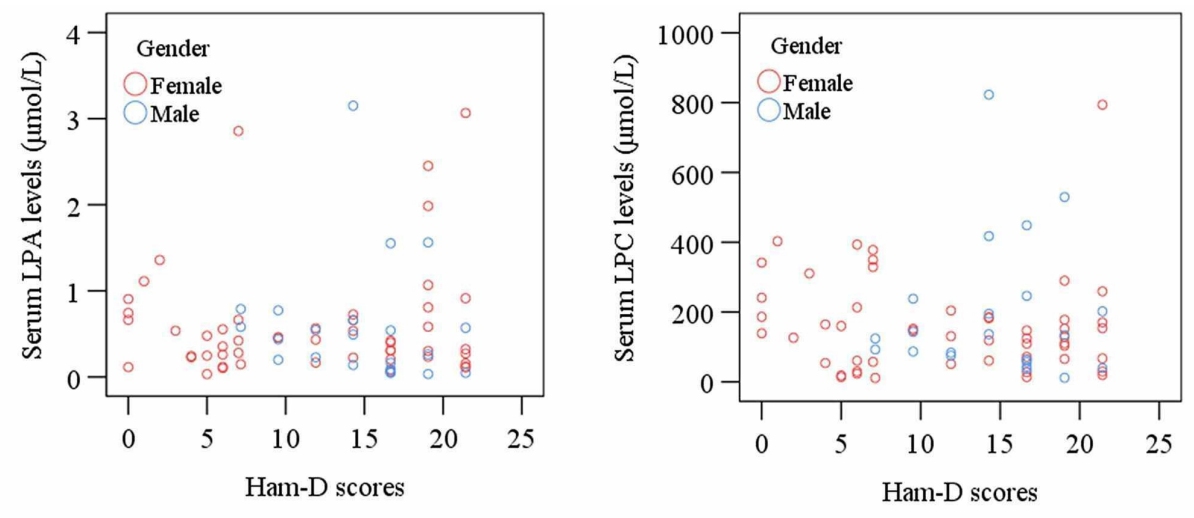

\section{FIGURE 2: Scatter plot indicates no significant correlations of serum LPA and LPC versus Ham-D scores in MDD patients}

LPA - lysophosphatidic acid; LPC - lysophosphatidylcholine; Ham-D - Hamilton depression rating scale; MDD - major depressive disorder

Significant positive correlations were observed between serum LPA and LPC levels in all MDD patients $(\mathrm{r}=0.739 ; \mathrm{p}<0.001)$, male MDD patients $(\mathrm{r}=0.937 ; \mathrm{p}<0.001)$, and female MDD patients $(\mathrm{r}=0.629 ; \mathrm{p}<0.001)$. The present study obtained no significant correlation between LPA or LPC levels and Ham-D scores.

\section{Discussion}

To the best of our knowledge, this is the first report concerning serum LPA and LPC in MDD patients from Bangladesh. The diagnosis of depression mainly depends on the structured clinical interview, and a reliable marker is not established yet for clinicians [17]. Here, we evaluated whether LPA or LPC levels could serve as an early risk assessment marker for MDD. Moreover, a study reported reduced serum autotaxin levels in MDD patients due to an impaired LPA axis function in the brain [26]. Liu et al. observed higher plasma LPC levels in MDD patients. Also, they found a significant positive correlation between these elevated LPC levels and the severity of depression [27]. Kim et al. also observed the decreased serum LPC levels in drug-treated or drug-naïve MDD patients compared to HCs [26]. A study reported lowered serum LPC levels in depression; others contrasted the assumption [26-29]. Thus, we verified the hypothesis that altered serum LPA and LPC levels are associated with the pathophysiology of MDD that could serve as early risk assessment or diagnostic purpose of depression. Despite the logic of our proposition, we observed that no significant alterations of serum LPA and LPC levels were happened in MDD patients compared to HCs, and that is why these phospholipids are unlikely to serve as early risk assessment markers of MDD. The present study is consistent with the past results where Gotoh et al. found no association of cerebrospinal fluid and plasma LPA levels with MDD [29]. The biological system obtained LPA from LPC with the enzyme autotoxin displays intriguing cell biology via $\mathrm{G}$ protein-coupled receptors. On the other hand, docosahexaenoic acid (DHA)containing LPC performs efficient transporter of its fatty acid into the brain crossing the blood-brain barrier with the help of major facilitator superfamily domain-containing $2 \mathrm{~A}$ receptor [30]. Along with the previous findings, the present study results assume that serum LPA or LPC levels could not be the applicant marker for MDD.

Finally, we measured correlation coefficients between serum LPA or LPC levels with patient characteristics and clinical severity scores of depressions. Here we observed no significant relationships between either serum LPA or LPC levels and patient profile such as age, gender, BMI, education, occupation, economic impression, and smoking history. We did not find any significant positive or negative correlation between serum LPA or LPC levels and the severity of depression. The present study results suggest that serum LPA or LPC levels would not play a significant role as a biomarker for clinical diagnosis of major depression.

This present study has a few limitations. The case-control study and the small sample size might also intervene with the findings. We didn't evaluate the food habit and treatment outcomes on the analyzed parameters. Therefore, we recommend further studies examining the correlation between LPA or LPC signaling systems to discover the role of phospholipids on the pathophysiology of depression.

\section{Conclusions}

The present study concluded that serum LPA and LPC levels would not serve as risk assessment markers for MDD. Serum levels of these lipids did not show any changes in MDD patients compared to HCs and also failed to show any correlation with the severity of depression. As a result, the present study suggests the association between serum LPA or serum LPC where the pathophysiology of depression is not well established. Therefore, this is a preliminary study. We recommend further researches to investigate the exact 
correlation between targeted glycerophospholipids and depression.

\section{Additional Information Disclosures}

Human subjects: Consent was obtained by all participants in this study. Ethical Review Committee of the Department of Psychiatry, Bangabandhu Sheikh Mujib Medical University (BSMMU), Dhaka, Bangladesh issued approval N/A. The study was conducted according to the world medical association declaration of Helsinki. Animal subjects: All authors have confirmed that this study did not involve animal subjects or tissue. Conflicts of interest: In compliance with the ICMJE uniform disclosure form, all authors declare the following: Payment/services info: All authors have declared that no financial support was received from any organization for the submitted work. Financial relationships: All authors have declared that they have no financial relationships at present or within the previous three years with any organizations that might have an interest in the submitted work. Other relationships: All authors have declared that there are no other relationships or activities that could appear to have influenced the submitted work.

\section{Acknowledgements}

We are thankful to the physicians and staff of the department of psychiatry, Bangabandhu Sheikh Mujib Medical University (BSMMU), Dhaka, Bangladesh, for their support in this study. We are also thankful to the participants of this study for their cooperation and participation.

\section{References}

1. Anjum S, Qusar MMAS, Shahriar M, Islam SMA, Bhuiyan MA, Islam MR: Altered serum interleukin-7 and interleukin-10 are associated with drug-free major depressive disorder. Ther Adv Psychopharmacol. 2020, $10.1177 / 2045125320916655$

2. Islam MR, Islam MR, Ahmed I, et al.: Elevated serum levels of malondialdehyde and cortisol are associated with major depressive disorder: a case-control study. SAGE Open Med. 2018, 10.1177/2050312118773953

3. Nishuty NL, Khandoker MMH, Karmoker JR, et al.: Evaluation of serum interleukin-6 and C-reactive protein levels in drug-naïve major depressive disorder patients. Cureus. 2019, 11:e3868. 10.7759/cureus.3868

4. Das R, Emon MPZ, Shahriar M, et al.: Higher levels of serum IL- $1 \beta$ and TNF- $\alpha$ are associated with an increased probability of major depressive disorder. Psychiatry Res. 2020, 10:113568. 10.1016/j.psychres.2020.113568

5. Islam MR, Ali S, Karmoker JR, et al.: Evaluation of serum amino acids and non-enzymatic antioxidants in drug-naïve first-episode major depressive disorder. BMC Psychiatry. 2020, 20:333. 10.1186/s12888-02002738-2

6. Islam MR, Islam MR, Shalahuddin Qusar MMA, et al.: Alterations of serum macro-minerals and trace elements are associated with major depressive disorder: a case-control study. BMC Psychiatry. 2018, 18:94. 10.1186/s12888-018-1685-z

7. Müller CP, Reichel M, Mühle C, et al.: Brain membrane lipids in major depression and anxiety disorders . Biochim Biophys Acta. 2015, 1851:1052-1065. 10.1016/j.bbalip.2014.12.014

8. Emon MPZ, Das R, Nishuty NL, et al.: Reduced serum BDNF levels are associated with the increased risk for developing MDD: a case-control study with or without antidepressant therapy. BMC Res Notes. 2020, 13:83. 10.1186/s13104-020-04952-3

9. Ali S, Nahar Z, Rahman MR, et al.: Serum insulin-like growth factor-1 and relaxin-3 are linked with major depressive disorder. Asian J Psychiatr. 2020, 53:102164. 10.1016/j.ajp.2020.102164

10. Daria S, Proma MA, Shahriar M, et al.: Serum interferon-gamma level is associated with drug-naïve major depressive disorder. SAGE Open Med. 2020, 10.1177/2050312120974169

11. Svennerholm L, Boström K, Jungbjer B, Olsson L: Membrane lipids of adult human brain: lipid composition of frontal and temporal lobe in subjects of age 20 to 100 years. J Neurochem. 1994, 63:1802-1811.

12. Rossy J, Ma Y, Gaus K: The organisation of the cell membrane: do proteins rule lipids? . Curr Opin Chem Biol. 2014, 20:54-59. 10.1016/j.cbpa.2014.04.009

13. Yung YC, Stoddard NC, Mirendil H, Chun J: Lysophosphatidic acid signaling in the nervous system. Neuron. 2015, 85:669-682. 10.1016/j.neuron.2015.01.009

14. Perrakis A, Moolenaar WH: Autotaxin: structure-function and signaling. J Lipid Res. 2014, 55:1010-1018. 10.1194/jlr.R046391

15. Aoki J, Inoue A, Okudaira S: Two pathways for lysophosphatidic acid production. Biochim Biophys Acta. 2008, 1781:513-518. 10.1016/j.bbalip.2008.06.005

16. Pagès $C$, Simon MF, Valet $P$, Saulnier-Blache JS: Lysophosphatidic acid synthesis and release. Prostaglandins Other Lipid Mediat. 2001, 64:1-10. 10.1016/s0090-6980(01)00110-1

17. Smith KM, Renshaw PF, Bilello J: The diagnosis of depression: current and emerging methods . Compr Psychiatry. 2013, 54:1-6. 10.1016/j.comppsych.2012.06.006

18. Aikawa S, Hashimoto T, Kano K, Aoki J: Lysophosphatidic acid as a lipid mediator with multiple biological actions. J Biochem. 2015, 157:81-9. 10.1093/jb/mvu077

19. Harrison SM, Reavill C, Brown G, et al.: LPA1 receptor-deficient mice have phenotypic changes observed in psychiatric disease. Mol Cell Neurosci. 2003, 24:1170-1179. 10.1016/j.mcn.2003.09.001

20. Law SH, Chan ML, Marathe GK, Parveen F, Chen CH, Ke LY: An updated review of lysophosphatidylcholine metabolism in human diseases. Int J Mol Sci. 2019, 20:1149. 10.3390/ijms20051149

21. Nakanaga K, Hama K, Aoki J: Autotaxin--an LPA producing enzyme with diverse functions . J Biochem. 2010, 148:13-24. 10.1093/jb/mvq052

22. Hu JS, Li YB, Wang JW, Sun L, Zhang GJ: Mechanism of lysophosphatidylcholine-induced lysosome 


\section{Cureus}

destabilization. J Membr Biol. 2007, 215:27-35. 10.1007/s00232-007-9002-7

23. Häkkinen T, Luoma JS, Hiltunen MO, et al.: Lipoprotein-associated phospholipase A(2), platelet-activating factor acetylhydrolase, is expressed by macrophages in human and rabbit atherosclerotic lesions.

Arterioscler Thromb Vasc Biol. 1999, 19:2909-2917. 10.1161/01.atv.19.12.2909

24. Carneiro AB, Iaciura BMF, Nohara LL, et al.: Lysophosphatidylcholine triggers TLR2- and TLR4-mediated signaling pathways but counteracts LPS-induced NO synthesis in peritoneal macrophages by inhibiting NFKB translocation and MAPK/ERK phosphorylation. PLoS One. 2013, 8:76233. 10.1371/journal.pone.0076233

25. Walther A, Cannistraci CV, Simons K, et al.: Lipidomics in major depressive disorder. Front Psychiatry. 2018, 9:459. 10.3389/fpsyt.2018.00459

26. Kim EY, Lee JW, Lee MY, et al.: Serum lipidomic analysis for the discovery of biomarkers for major depressive disorder in drug-free patients. Psychiatry Res. 2018, 265:174-182. 10.1016/i.psychres.2018.04.029

27. Liu X, Li J, Zheng P, et al.: Plasma lipidomics reveals potential lipid markers of major depressive disorder. Anal Bioanal Chem. 2016, 408:6497-6507. 10.1007/s00216-016-9768-5

28. Seretti A, Cusin C, Lattuada E, Di Bella D, Catalano M, Smeraldi E: Serotonin transporter gene (5-HTTLPR) is not associated with depressive symptomatology in mood disorders. Mol Psychiatry. 1999, 4:280-283. 10.1038/sj.mp.4000485

29. Gotoh L, Yamada M, Hattori K, et al.: Lysophosphatidic acid levels in cerebrospinal fluid and plasma samples in patients with major depressive disorder. Heliyon. 2019, 5:E01699. 10.1016/j.heliyon.2019.e01699

30. Lagarde M, Bernoud N, Brossard N, et al.: Lysophosphatidylcholine as a preferred carrier form of docosahexaenoic acid to the brain. J Mol Neurosci. 2001, 16:2-3. 10.1385/JMN:16:2-3:201 